\title{
Tandem bullet injury: An unusual variant of an unusual injury
}

\author{
L. du Toit-Prinsloo ${ }^{*}{ }^{-}$, N. K. Morris ${ }^{1}$ and G. Saayman ${ }^{1}$ \\ ${ }^{1}$ Department of Forensic Medicine, University of Pretoria, Private Bag X323, Arcadia, 0007, South \\ Africa
}

*L. du Toit-Prinsloo

Email: lorraine.dutoit@up.ac.za

\section{Case report}

A 41-year old male sustained a fatal gunshot wound during a house robbery. Police enquiries and scene investigation revealed that a single shot had been fired and that one spent $9 \mathrm{~mm}$ cartridge case was retrieved from the scene.

At autopsy the beige short sleeved jacket and printed white T-shirt worn by the deceased each displayed a single perforating defect on the right front aspect (approximately overlying the pectoral region), with associated blood staining (Figure 1).

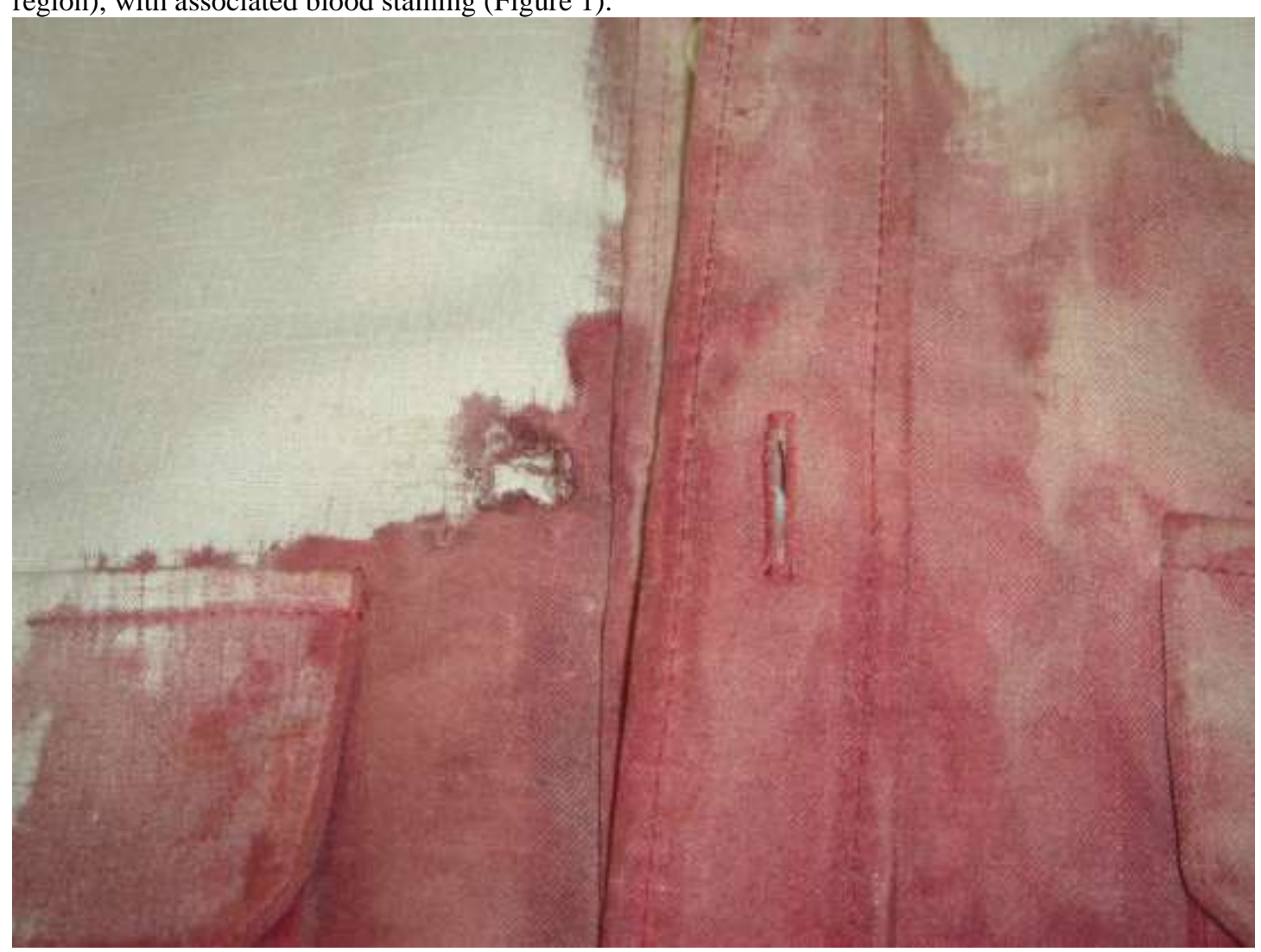

Fig. 1 : Outer shirt of the deceased, displaying penetrating defect on the front

Two penetrating wounds were present on the skin of the anterior aspect of the right pectoral region, one above the other and in close proximity to each other, located approximately $6 \mathrm{~cm}$ medial to the right nipple (Figure 2). The uppermost wound was round in shape, measuring $0,9 \mathrm{~cm}$ in diameter with a distinct but irregular concentric peripheral collar of abrasion. The lowermost wound was oval in shape, measuring $2 \times 1 \mathrm{~cm}$ in size and with a relatively "clean cut" appearance of the edges, except in the 9 o'clock to 1 o'clock position, where there was irregular superficial abrasion of the adjacent skin. Neither of the wounds displayed features of associated heat injury, soot deposition or powder tattooing. 


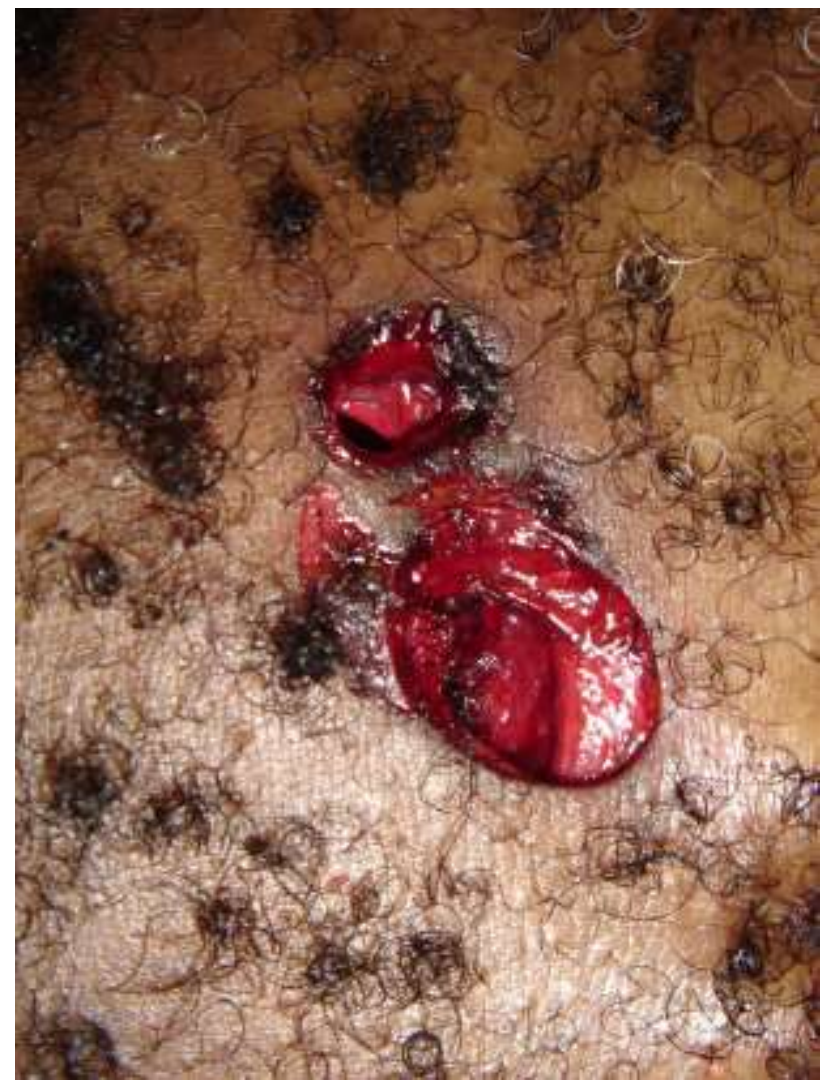

Fig. 2 : Close up view of skin wounds in right parasternal position

Upon palpation and superficial dissection of the back, at a point $25 \mathrm{~cm}$ below the level of the shoulder and $5 \mathrm{~cm}$ medial to the midline on the left, a somewhat misshapen $7,65 \mathrm{~mm}$ projectile was recovered. Transillumination (C-arm fluoroscopy) revealed the presence of two further foreign metal objects in the chest region; one had the typical appearance of a heavy calibre handgun projectile (left posterior chest wall), whilst the other had the appearance of a spent (misshapen) cartridge case, in the right chest cavity (Figure 3).

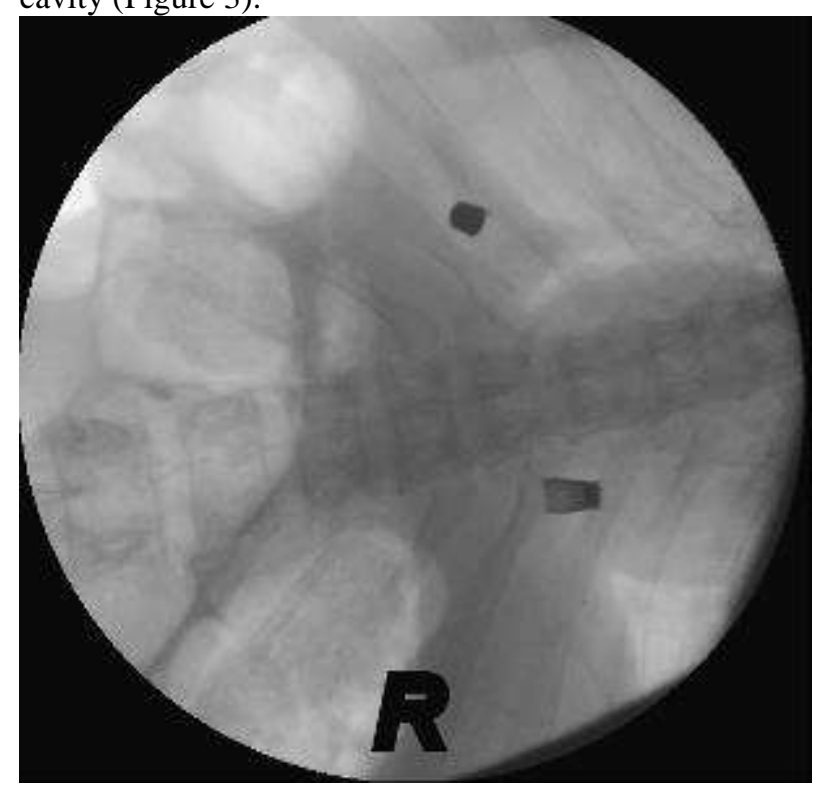

Fig. 3 : Radiographic image of spent $9 \mathrm{~mm}$ projectile (upper) and $7.65 \mathrm{~mm}$ cartridge case (lower) in chest cavity of deceased

Upon opening of the chest, a free-lying deformed 7,65mm cartridge case was recovered from the right pleural cavity, with bilateral hemothoraces. There were two penetrating wound tracts traversing the right anterior chest wall between the third and fourth ribs parasternally (with some fragmentation of the adjacent sternum) and with two slightly divergent wound tracts subsequently perforating the chest cavity from front to back and from right to left. One tract tangentially involved (inter alia) the 
anteromedial portion of the upper lobe of the right lung, the proximal ascending aorta and the posteromedial portion of the lower lobe of the left lung, culminating where the spent $7,65 \mathrm{~mm}$ calibre projectile had been removed from the superficial subcutaneous tissues of the back (as described above). The second wound tract traversed the anterior pericardial sac, the right and left atria of the heart and the posteromedial portion of the lower lobe of the left lung, culminating in the eighth intercostal space (posterior), where a spent $9 \mathrm{~mm}$ calibre projectile was found. Interestingly, the front end of the latter projectile had a clearly visible indentation, being a mirror imprint which corresponded to the base of the cartridge case of the $7,65 \mathrm{~mm}$ projectile (Figures 4 and 5).

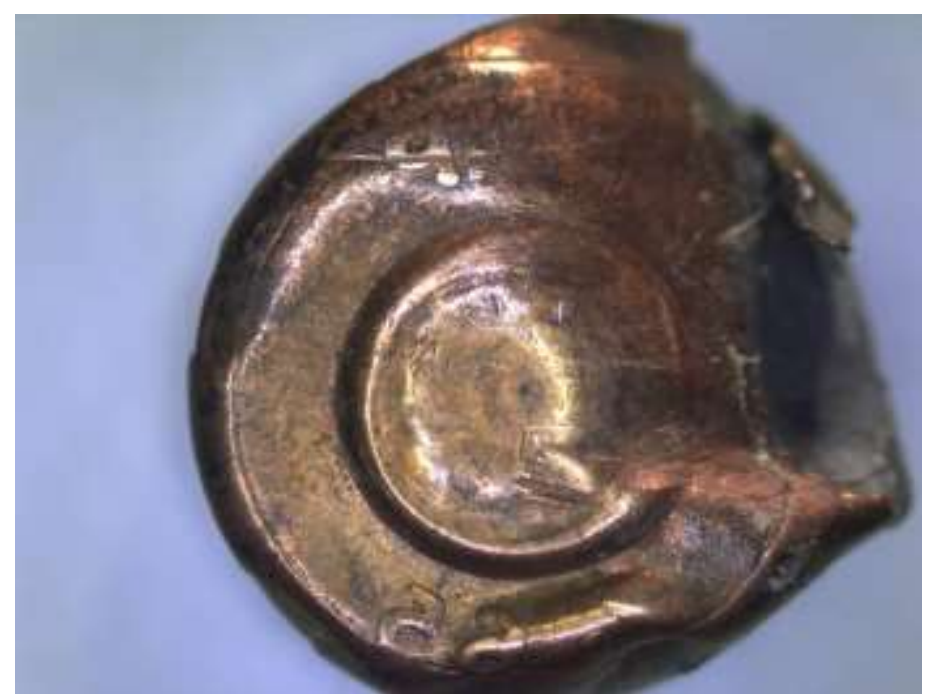

Fig. 4 : Front end ("nose") of spent $9 \mathrm{~mm}$ projectile, recovered from posterior chest wall

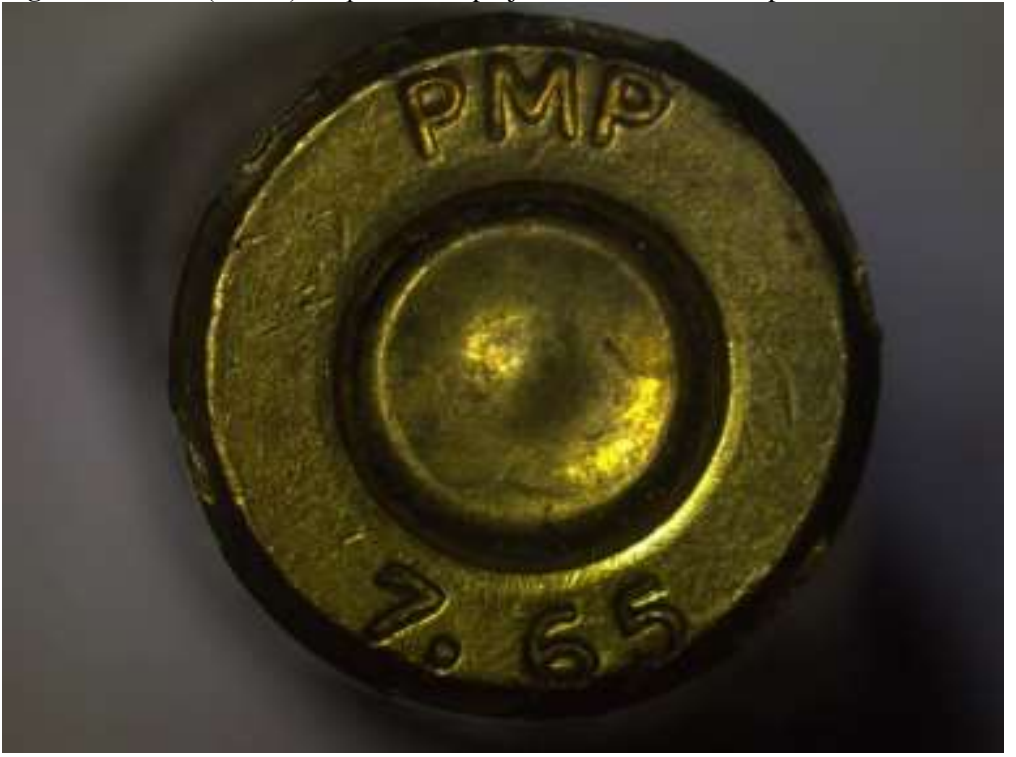

Fig. 5 : Base of $7.65 \mathrm{~mm}$ cartridge case, recovered from pleural cavity

Based on the overall findings, we postulate that the intact $7,65 \mathrm{~mm}$ round had previously lodged within the barrel of the firearm, where it was subsequently impacted from behind by the fired $9 \mathrm{~mm}$ round and with all the projectiles then entering the chest of the deceased. The approximate configuration of the projectiles would then have been as displayed in the reconstructed sequence shown in Figure 6 . It is not certain which of the wounds was caused by which of the projectiles, but it seems likely that the larger, ovoid wound may have been caused by the $7,65 \mathrm{~mm}$ cartridge and/or projectile, with the $9 \mathrm{~mm}$ projectile having caused the uppermost of the two (skin) defects. Subsequent ballistic examination of the projectiles revealed no features to suggest that the $7,65 \mathrm{~mm}$ round had indeed undergone combustion of the primer or propellant, although the two components may have physically separated before entering the body of the victim. 


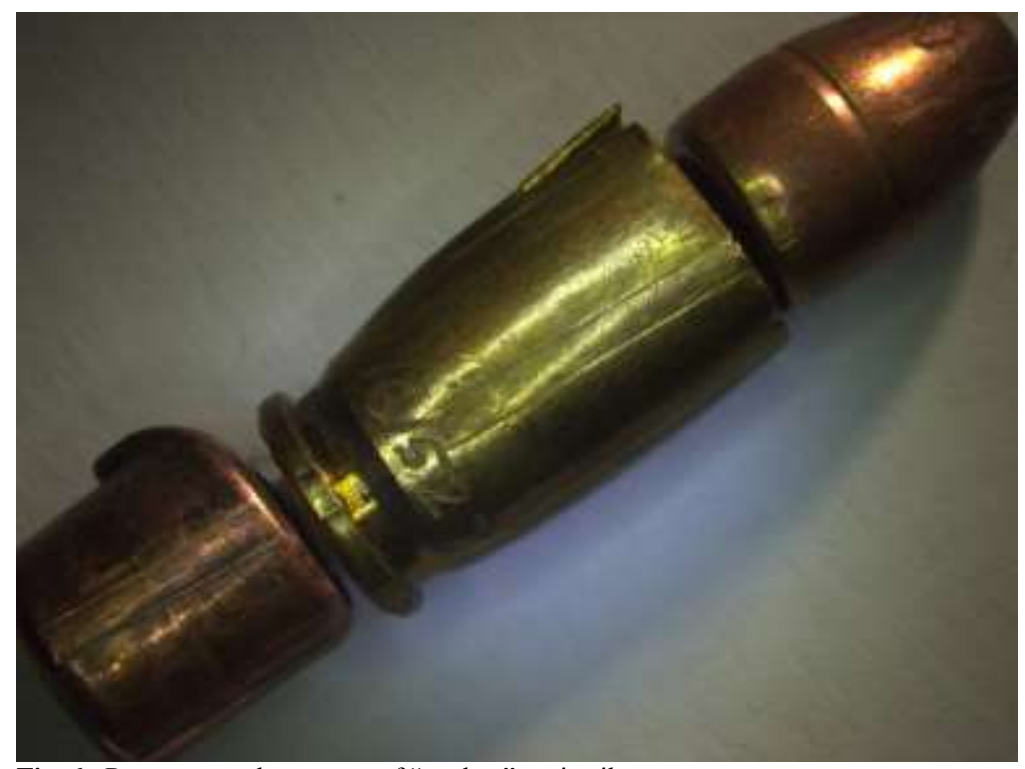

Fig. 6 : Reconstructed sequence of "tandem" projectiles

\section{Discussion}

The term "tandem bullets" is used to refer to the phenomenon of multiple projectiles being simultaneously discharged from a firearm [1,2]. The term "piggyback bullets" has also been used in this regard [1]. On rare occasions, a projectile (or other foreign object) may remain lodged within the barrel of a firearm, for a variety of possible reasons. With subsequent further discharge, serious damage may take place (such as rupture of the barrel) or both projectiles can be propelled from the barrel. These projectiles usually demonstrate unstable flight ballistics [3] and can result in a multiplicity of injury outcomes on the bodies of victims, including multiple and irregular entrance gunshot wounds, which may complicate the interpretation of findings and impact on subsequent legal proceedings $[1,4]$.

Review of the literature suggests that this is a relatively scarcely reported phenomenon, with the first report thereof apparently being that of Timperman and Cnops, in 1975 [2]. It occurs "when one bullet or other foreign body becomes lodged inside the barrel and receives a "kick" on its base by the nose of the next fired bullet" [5]. The obstruction may result from either insufficient quality or quantity of propellant in the cartridge case of a round of ammunition, or with incomplete combustion of the propellant [1].

An even rarer variation on the cause of the obstruction in the barrel of the firearm includes the use of incorrect calibre ammunition (usually smaller calibre ammunition), which may result in the entire round (including cartridge case) being pushed down the barrel $[1,5,6]$. Examples have been documented where .32 calibre rounds have been driven into the barrels of .38 calibre firearms $[5,6]$, as was first reported by Mollan and Beavis [6]. In our case, an intact 7,65mm round had become lodged within the barrel of a 9mm semi-automatic pistol, "piggy-backing" the $9 \mathrm{~mm}$ round into the victim.

This phenomenon has also been described with respect to a shotgun [7], where fragments of a 20 gauge shotgun shell and a 12 gauge wadding was recovered from the victim. Another variation involves the inadvertent discharge of foreign objects of a non-ballistic nature: Ellis reports a suicidal gunshot wound to the head where a cleaning brush had been left inside the barrel of the firearm - both the cleaning brush and bullet were found in the head of the victim [8].

Gunshot wounds - both entrance and exit in nature - may display a wide variation in morphological features. Atypical presentations may result in inexperienced or inattentive examiners misinterpreting findings at autopsy. The similarity of appearances between, for example, entrance wounds caused by unstable or misshapen projectiles and those of shored exit wounds, may have serious implications for parties implicated in such shootings. Similarly, failure to appreciate that a single discharge of a firearm may (albeit rarely) result in multiple (two, or even three) entrance wounds - some of which may have atypical features - may also result in miscarriages of justice. An example may be where accidental 
discharge of a weapon results in multiple entrance wounds, prosecuting authorities may be reluctant to accept an explanation suggesting that a single shot had been fired.

South Africa has a very high incidence of firearm related deaths, reported to be 26.8 per 100000 and ranking third highest in the world [9]. Accurate statistics are not available, but it has been reported that there is an estimated 8 million legal and illegal firearms in South Africa and that approximately fifty percent of homicides in South African cities are due to firearms [10]. Where such high rates of firearm related injuries and fatalities are encountered, it is important for clinicians and forensic pathologists to be aware of the spectrum of both typical and atypical or unusual ballistic injuries which may be encountered. Such awareness may prompt greater accuracy of observation and collaboration with ballistic experts, which may in turn impact both on clinical treatment decisions and on subsequent legal proceedings.

In conclusion, tandem bullet injuries are relatively rare but the unusual features may have important implications, both with respect to treatment of victims and for subsequent legal proceedings. In communities where firearm wounding is common, clinicians and forensic pathologists should be mindful of all the unusual and atypical forms of presentation which may be seen in relation to gunshot wounds. Thorough initial examination (including where possible, the use of transillumination or radiographic imaging), as well as subsequent examination (and photography) by ballistic experts, may be of great importance in ensuring that the judicial process is correctly served.

\section{Acknowledgments}

The authors would like to thank dr CM Cronjé and ms E Coetzee for their assistance with the taking of photographs.

\section{References}

1. Di Maio VJM. Gunshot wounds Practical aspects of firearms, ballistics, and forensic techniques. 2nd ed. CRC Press, Boca Raton, London, New York, Washington DC; 1999. p292.

2. Timperman J, Cnops L. Tandem bullet in the head in a case of suicide. Med Sci Law. 1975;15:280-3.

3. Sinha JK, Bhattacharyya CN. Identification of tandem Bullets. Med Sci Law. 1989; 29(3):249-50.

4. Hiss J, Kahana T. Confusing exit gunshot wound - "Two for the price of one". Int J Legal Med. 2002; 116:47-9

5. Lilienstein DA and Van Gelder CM. A mystery: One wound, multiple bullets. J Forensic Leg Med. 2008;15: 343-5.

6. Mollan RAB, Beavis V. A curious gunshot injury. Injury. 1978;9(4): 327-328.

7. Simmons GT. Findings in gunshot wounds from tandem projectiles. J Forensic Sci. 1997;42(4):678-81.

8. Ellis PS. Fatal gunshot injury caused by an unusual projectile - a barrel-cleaning brush as a tandem bullet. Am J Forensic Med Pathol 1997;18(2):168-71.

9. Abrahams N. Jewkes R and Mathews A. Guns and gender-based violence in South Africa. S Afr Med J. 2010; 100(9):586-8.

10. Chamisa I. Civilian abdominal gunshot wounds in Durban, South Africa: A prospective study of 78 cases. Ann R Coll Surg Engl. 2008;90(7):581-6. 